Einführung zum Thema

Pneumologe 2021 · 18:209-211

https://doi.org/10.1007/s10405-021-00393-2

Angenommen: 24. März 2021

(c) Springer Medizin Verlag $\mathrm{GmbH}$, ein Teil von Springer Nature 2021

\section{Redaktion}

C. Vogelmeier, Marburg

B. Schmeck, Marburg

\author{
Claus Vogelmeier ${ }^{1,2} \cdot$ Bernd Schmeck $^{1,2,3,4}$ \\ ${ }^{1}$ Klinik für Innere Medizin, Schwerpunkt Pneumologie, Philipps-Universität Marburg, Marburg, \\ Deutschland \\ ${ }^{2}$ Institut für Lungenforschung, Philipps-Universität Marburg, Marburg, Deutschland \\ ${ }^{3}$ Deutsches Zentrum für Lungenforschung, Standortverbund UGMLC (Universities of Giessen and Marburg \\ Lung Center), Deutschland, Marburg, Deutschland \\ ${ }^{4}$ Deutsches Zentrum für Infektionsforschung, Standort Gießen-Marburg-Langen, Marburg, Deutschland
}

\title{
Genetik von Lungenkrankheiten
}

Erkrankungen der Atemwege und des Lungenparenchyms gehören weltweit zu den hinsichtlich Prävalenz, Morbidität und Mortalität wichtigsten Erkrankungen. Oft konzentrieren sich die klinischen und pathophysiologischen Betrachtungen auf exogene Auslöser, z. B. Infektionserreger, Allergene oder toxische Substanzen. Jedoch steht einer oftmals groBen Gruppe von Exponierten nur eine begrenzte Zahl tatsächlich Erkranktergegenüber. Dies legt einen bedeutsamen Einfluss endogener, unter anderem genetischer Ursachen nahe.

\section{》) Vielen Lungenerkrankungen liegen neben exogenen Faktoren auch genetische Ursachen zugrunde}

In diesem Heft stellen daher klinisch und grundlagenwissenschaftlich erfahrene Kolleginnen und Kollegen die Bedeutung genetischer Ursachen für wichtige Lungenkrankheiten dar und beschreiben die Implikationen für die Patientenbehandlung:

Lungenentzündungen sind die weltweit häufigste Todesursache durch Infektionskrankheiten. Gerade erleben wir mit COVID-19 die enormen medizinischen, ökonomischen und gesellschaftlichen Auswirkungen einer pandemischen Pneumonie in Form einer ,emerging infectious disease“. Der Fokus liegt bei diesen Erkrankungen ganz offensichtlich auf dem infektiösen Erreger, sogar in der Nomenklatur (Pneumokokkenpneumonie etc.), v. a. da er Adressat sehr erfolgrei- cher Präventions- (Impfungen) und Therapiemaßnahmen (Antibiotika) ist. Neue molekularbiologische Methoden und genetische Erkenntnisse demonstrieren jedoch bedeutende mono- oder polygenetische Einflüsse auf die Suszeptibilität für eine Pneumonie und deren Verlauf (Beitrag Bertrams et al.). Die Konsequenzen für eine entsprechend personalisierte Infektionsmedizin müssen erst in Studien und für die Praxis entwickelt werden.

Asthma bronchiale ist eine chronisch entzündliche Erkrankung der Atemwege, die häufig schon im Kindesalter symptomatisch wird. Sie zeigt eine große klinische und immunologische Heterogenität. In der aktuellen medizinischen Betrachtung stehen auch hier Umweltfaktoren im Vordergrund, v. a. Allergien, aber auch Medikamente und physikalische Reize. Durch Kohortenstudien, Sequenzierungsansätze, aber auch experimentelle Untersuchungen in Modellorganismen wurden Punktmutationen identifiziert, die das individuelle Risiko, an Asthma zu erkranken, aber auch das Ansprechen auf Therapien (Beitrag Borchers et al.) mit beeinflussen. Ebenfalls von Bedeutung sind Veränderungen im Epigenom, also nicht der Basensequenz, die ihrerseits durch die Umwelt - teils schon vorgeburtlich - geprägt werden.

Die COPD („chronic obstructive pulmonary disease") ist eine chronisch entzündliche Erkrankung der Atemwege und des Lungenparenchyms. Sie wird durch inhalative Noxen, v.a. Zigarettenrauch, aber auch andere Umweltexpositionen ausgelöst. Sie tritt daher üblicherweise erst in höherem Erwachsenenalter klinisch in Erscheinung und 
ist in der Regel mit einer Vielzahl von Komorbiditäten (kardial, metabolisch ...) vergesellschaftet. In den letzten Jahren wurden genetische Assoziationen für verschiedene klinische Manifestationen (Bronchitis, Emphysem, Komorbiditäten), Pathophysiologien (oxidativer Stress, Proteasenaktivität, Inflammation) und das Therapieansprechen identifiziert (Beitrag Jarosch et al.). Eine besondere Form ist der $\alpha_{1}$-Antitrypsinmangel, eine monogenetische Ursache, die zum Teil zu direkten Therapiekonsequenzen führt und auf die daher unbedingt getestet werde sollte.

Die interstitiellen Lungenkrankheiten und speziell die idiopathische pulmonale Fibrose stellen trotz neu zugelassener Therapiemöglichkeiten weiterhin große klinische Herausforderungen dar. Neben äußeren Einflüssen auf den Krankheitsverlauf wurden schon früh familiäre Krankheitscluster identifiziert, die genetische Ursachen nahelegten. Die inzwischen identifizierten genetischen Veränderungen finden sich jedoch teilweise sowohl bei den familiären wie bei den sporadischen Krankheitsformen (Beitrag Markart et al.). Zusammenfassend liegen also vielen Lungenerkrankungen neben exogenen Faktoren auch genetische Ursachen zugrunde. Sie bieten zukünftig die Chance einer besseren individuellen Risikoabschätzung und/oder zielgerichteteren Therapie. In ausgewählten Fällen sind jedoch auch heute schon gezielte genetische Testungen indiziert.

Prof. Dr. Claus Vogelmeier,

Prof. Dr. Bernd Schmeck

\section{Korrespondenzadresse}

Prof. Dr. Bernd Schmeck

Klinik für Innere Medizin, Schwerpunkt Pneumologie, Philipps-Universität Marburg Hans-Meerwein-Str. 2, 35043 Marburg, Deutschland

bernd.schmeck@uni-marburg.de

Interessenkonflikt. C. Vogelmeier und B. Schmeck geben an, dass kein Interessenkonflikt besteht.

\section{Julius-Springer-Preis für Pneumologie 2021 verliehen}

Springer Medizin würdigt PD Dr. Matthias Held für herausragenden Beitrag

Eine akute Lungenembolie kann lebensbedrohlich sein. Nicht weniger gefährlich sind jedoch chronisch geprägte Embolien, die Behandelnde in der ärztlichen Nachsorge häufig vor Herausforderungen stellen. Für seinen Beitrag Nachsorge bei Lungenembolie - was ist empfehlenswert? erschienen in Der Pneumologe Heft 5 | 2019 erhält PD

Dr. Matthias Held als erstgenannter Autor den Julius-Springer-Preis für Pneumologie 2021. Die Preisverleihung fand im Rahmen einer Sitzung der Herausgeber*innen von Der Pneumologe Ende Juni statt. Der Preis ist mit $2.500 €$ dotiert und wird alle zwei Jahre vergeben.

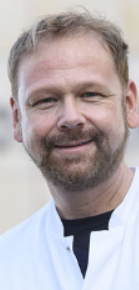

Bild: Preisträger des Julius Springer Preis Pneumologie | PD Dr. Matthias Held @ Daniel Peter, Würzburg

Der Preisträger und seine Koautor*innen, Dr. Annabel Plaßmeier und Hubertus Rolke, erklären in ihrem Artikel eingängig, wie eine gezielte Nachsorge dazu dienen kann, das Rückfallrisiko für Lungenembolien zu vermindern, nicht komplett aufgelöste Tromben zu erkennen und Begleiterkrankungen auf die Spur zu kommen. Folgeschäden würden dadurch so gering wie möglich gehalten oder sogar vermieden werden.

Die Autor*innen erläutern kompakt, welche Kernpunkte in der Nachsorge zu beachten sind und wie Belastungseinschränkungen - eine besonders häufige Folge von Lungenembolien - abgeklärt werden können. Als weiterer Eckpfeiler der Nachsorge wird die sorgfältige Abwägung der Behandlung mit gerinnungshemmenden Medikamenten aufgeführt. Denn der Einsatz solcher Medikamente zur Vermeidung eines Rückfalls sollte immer mit dem individuellen Blutungsrisiko abgewogen werden. Da Lungenembolien auch auf versteckte Tumorerkrankungen hinweisen können, schließt der Beitrag mit Untersuchungsmöglichkeiten zum Aufspüren von Tumoren.

„Die Nachsorge nach Lungenembolie ist ein vielschichtiges Thema, bei dem komplexe Zusammenhänge gleichzeitig bedacht werden müssen. Der Beitrag Nachsorge bei Lungenembolie - was ist empfehlenswert? von Held und Koautor*innen hat es verstanden, dies wissenschaftlich fundiert und gleichzeitig leicht verständlich darzustellen", so Prof. Dr. Horst Olschewski, Herausgeber von Der Pneumologe und Jurymitglied.

Prof. Dr. Heinrich Worth, Schriftleiter von Der Pneumologe und ebenfalls Jurymitglied, ergänzt: „In der vorliegenden Arbeit werden didaktisch hervorragend aufbereitete Informationen über die Nachsorge nach Lungenembolie verbunden mit der Darstellung eines strukturierten, vom Autor selbst entwickelten und evaluierten Programms, das in der klinischen Praxis eine Versorgungslücke schließen kann."

Die drei Jurymitglieder, Prof. Dr. Daniela Gompelmann, ebenfalls Herausgeberin von Der Pneumologe, Prof. Dr. Horst Olschewski und Prof. Dr. Heinrich Worth kürten den Beitrag nach Aktualität, Fortbildungswert, Darstellung und Didaktik. Insgesamt 53 Beiträge standen zur Auswahl.

PD Dr. Matthias Held ist Facharzt für Innere Medizin mit den Schwerpunkten Pneumologie, Kardiologie und Notfallmedizin. Während seiner Tätigkeit als Oberarzt an der Missioklinik in Würzburg war er am Aufbau des Zentrums für pulmonale Hypertonie und Lungengefäßerkrankungen beteiligt. Seit 2018 ist er Chefarzt der Medizinischen Klinik mit Schwerpunkt Pneumologie und Beatmungsmedizin an der Missioklinik Würzburg. Seit Oktober 2020 ist er zudem Ärztlicher Direktor am Klinikum Würzburg Mitte.

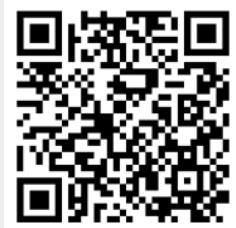

Hier geht es direkt zum gekürten Beitrag! 
Hier steht eine Anzeige.

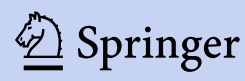

